\title{
Associative Self-Anchoring Interacts with Obtainability of Chosen Objects
}

\author{
Charlotte Prévost *, Niall Bolger and Dean Mobbs* \\ Department of Psychology, Columbia University, New York, NY, USA
}

While there is evidence that implicit self-esteem transfers to chosen objects (associative self-anchoring), it is still unknown whether this phenomenon extends to explicit selfesteem. Moreover, whether the knowledge that these objects might belong to the self in the future or not affects the evaluation of these objects has received little attention. Here, we demonstrate that evaluations of chosen objects are further enhanced when they are obtainable as compared to when they are not in participants with high explicit selfesteem, whereas participants with low explicit self-esteem exhibit the opposite pattern. These findings extend previous results and shed new light on the role of self-esteem in altering preferences for chosen objects depending on their obtainability.

\section{OPEN ACCESS}

Edited by:

Seung-Lark Lim,

University of Missouri - Kansas City,

USA

Reviewed by:

Raphael Koster,

University College London, UK

Amitai Shenhav,

Princeton University, USA

*Correspondence:

Charlotte Prévost

charlotte.prevost@unige.ch;

Dean Mobbs

dm2912@columbia.edu

Specialty section:

This article was submitted to

Emotion Science,

a section of the journal

Frontiers in Psychology

Received: 28 August 2015 Accepted: 16 December 2015

Published: 11 January 2016

Citation:

Prévost $C$, Bolger $N$ and Mobbs $D$ (2016) Associative Self-Anchoring

Interacts with Obtainability of Chosen

Objects. Front. Psychol. 6:2012. doi: 10.3389/fpsyg.2015.02012
Keywords: self-esteem, associative self-anchoring, psychological reactance, decision-making

\section{INTRODUCTION}

Self-esteem promotes 'positive illusions' such as overly positive self-evaluations, exaggerated perceptions of control and unrealistic optimism; resulting in increased well-being and mental health (Taylor and Brown, 1988). Consistent with the idea that people with a higher sense of selfesteem are better at deceiving themselves, self-esteem has also been suggested to play a key role in the enhancement of valuation of chosen objects. For instance, it has been shown that when choosing an object, an association between the self and the object is formed, a phenomenon referred to as associative self-anchoring (Cadinu and Rothbart, 1996; Gawronski et al., 2007). Consequently, evaluations of the self transfer to the chosen object such that the evaluation of the chosen object is modulated by implicit self-esteem rather than cognitive dissonance (Gawronski and Strack, 2004; Gawronski et al., 2007). For instance, Gawronski et al. (2007) asked participants to make implicit evaluations of two pictures before choosing between them, and showed that implicit evaluations of the chosen picture depend on implicit evaluations of the self. Implicit self-esteem is commonly measured using an initials preference task where participants are asked to rate the likeability of all letters of the alphabet presented in a random order on a 1-5 Likert scale. The preference for one's own initials is subsequently interpreted as an index of implicit self-esteem. Conversely, explicit self-esteem is measured using questionnaires asking participants to directly reflect on their self-evaluation (e.g., by indicating on a 1-7 Likert scale how much they agree with the following statement: "I have high self-esteem"). Thus, while implicit self-esteem refers to an automatic or unconscious self-evaluation, explicit self-esteem refers to a more conscious and reflective evaluation. Yet, it is still unknown whether associative self-anchoring extends to explicit self-esteem.

Low self-esteem has been associated with an increased tendency to exhibit reactant behavior in the case of freedom elimination (Joubert, 1990). According to psychological reactance theory, eliminating a behavioral freedom previously established will induce a motivational state aimed at restoring the lost freedom, such that a chosen object no longer obtainable will be perceived as more 
attractive (Brehm, 1966; Brehm and Brehm, 1981). However, whether self-esteem differentially affects the evaluation of chosen objects based on the opportunity of actually receiving these objects has remained untested.

Drawing on the associative self-anchoring account and psychological reactance theory, we hypothesized that people with a high sense of explicit self-esteem would exhibit enhanced evaluations for chosen objects that they might obtain compared to chosen objects that are not obtainable, whereas people with low explicit self-esteem would exhibit enhanced evaluations for chosen objects that are not obtainable compared to chosen objects that are obtainable. To test these predictions, we designed a paradigm where participants were asked to indicate relative evaluations for one of two objects presented side-by-side before and after choosing one of them in conditions where chosen objects were obtainable (i.e., one of the chosen object would randomly be selected by the computer and given to them at the end of the experiment) or not (Figure 1).

\section{MATERIALS AND METHODS}

\section{Participants}

Twenty-eight Columbia University students (15 females) with a mean age of $22.71 \pm 4.40$ participated in the study. All participants were free of neurological or psychiatric disorders and had normal or correct-to-normal vision. Written informed consent was obtained from all participants, and the study was approved by the Columbia University research ethics committee.

\section{Experimental Procedure}

The experiment was performed simultaneously by two gendermatched participants in two separate testing rooms. Upon arrival, participants were told that they would be administered the same task at the same time. The experiment included five different tasks and lasted approximately $1 \mathrm{~h}$, followed by a short post-experiment questionnaire and the single item selfesteem questionnaire (Robins et al., 2001). In this questionnaire, participants had to indicate by moving a cursor along a 1-7 Likert scale (increments of 1) to what extent the statement "I have high self-esteem" was true of them (mean score $=5.28 \pm 1.06$ ).

Stimuli consisted of 280 objects from the Columbia University bookstore, which were presented in pairs, two objects at a time side-by-side (Figure 1).

\section{Evaluation Task 1}

To assess participants' relative evaluations of all 140 pairs of objects, we asked them to imagine how much they would prefer to obtain each object relative to the other object at the end of the experiment (Figure 1A). These evaluations were relative in that increasing the evaluation of one object (i.e., by adding more stars) automatically decreased the evaluation of the other object (i.e., by removing stars). Participants were instructed about the meaning of each star combination, namely: three stars for each object indicated an equal preference, 2.5 stars for one of the objects versus 3.5 stars for the other object indicated a moderate preference, 2 stars versus 4 stars indicated a strong preference for one of the objects over the other object, 1.5 stars versus 4.5 stars indicated a very strong preference and 1 star versus 5 stars indicated an extremely strong preference. Evaluations were made in a self-paced manner, separated by an inter-trial interval (ITI) of 1-5 s. All pairs of items were assigned randomly across subjects. Each item was only presented once.

The purpose for Evaluation task 1 was twofold. First, it provided us with relative evaluations for each pair of objects, and second, it allowed us to extract only those pairs of objects for which a change in evaluation could be optimally detected. Indeed, pairs of objects for which one of the two objects was very strongly to extremely strongly preferred (i.e., 1.5 versus 4.5 stars, or 1 versus 5 stars) were not optimized to allow for an evaluation enhancement of the favored object because of a ceiling effect, while pairs of objects with equal evaluations (i.e., three stars for each object) were not optimal to trigger reactant behavior, which is expressed by an increase in the attractiveness of the unobtainable alternative proportional to its original relative evaluation (Brehm, 1966). Thus, 80 pairs of objects for which one of the two objects was moderately to strongly preferred (i.e., 2.5 versus 3.5 stars, or 2 versus 4 stars) were selected and included in subsequent tasks. If 80 pairs of objects with these combinations were not available for any given participant, we selected the number of pairs with these combinations that were available, using a criterion of a minimum of 50 pairs (note that all participants met this criterion). On average, participants were presented with $64.61 \pm 13.64$ choice pairs (median $=64)$.

The rationale for having participants make initial preference ratings between two items presented at a time rather than have them rate each item independently as is usually done in cognitive dissonance experiments (Sharot et al., 2009, 2012; Izuma et al., 2010; Jarcho et al., 2011; Qin et al., 2011; Kitayama et al., 2013) was to avoid the possible confounds raised by Chen and Risen (Chen and Risen, 2010; Izuma and Murayama, 2013). Indeed, in the conventional "Free Choice Paradigm," participants are first asked to provide individual ratings for a number of items. They are then asked to make choices between two items that have either similar ratings ("difficult choices") or significantly different ratings ("easy choices"), before re-evaluating each of these items separately. A critical issue associated with this paradigm is that the two items previously rated independently are then evaluated side-by-side at the time of choice, which provides additional information about true preference, and bias measured attitude change following choices. Accordingly, it was shown that the spread of alternative observed following difficult choices could occur in the absence of a preference change and thus, without experiencing dissonance (Chen and Risen, 2010; Izuma and Murayama, 2013). Note that Izuma and Murayama (2013) demonstrated that choices involving differently valued alternatives were not affected by spurious dissonance effects in this paradigm. Thus, in the current experiment, possible confounds were avoided by (1) measuring true preference by having participants evaluate the items relative to one another and (2) making choices between differently valued alternatives. 


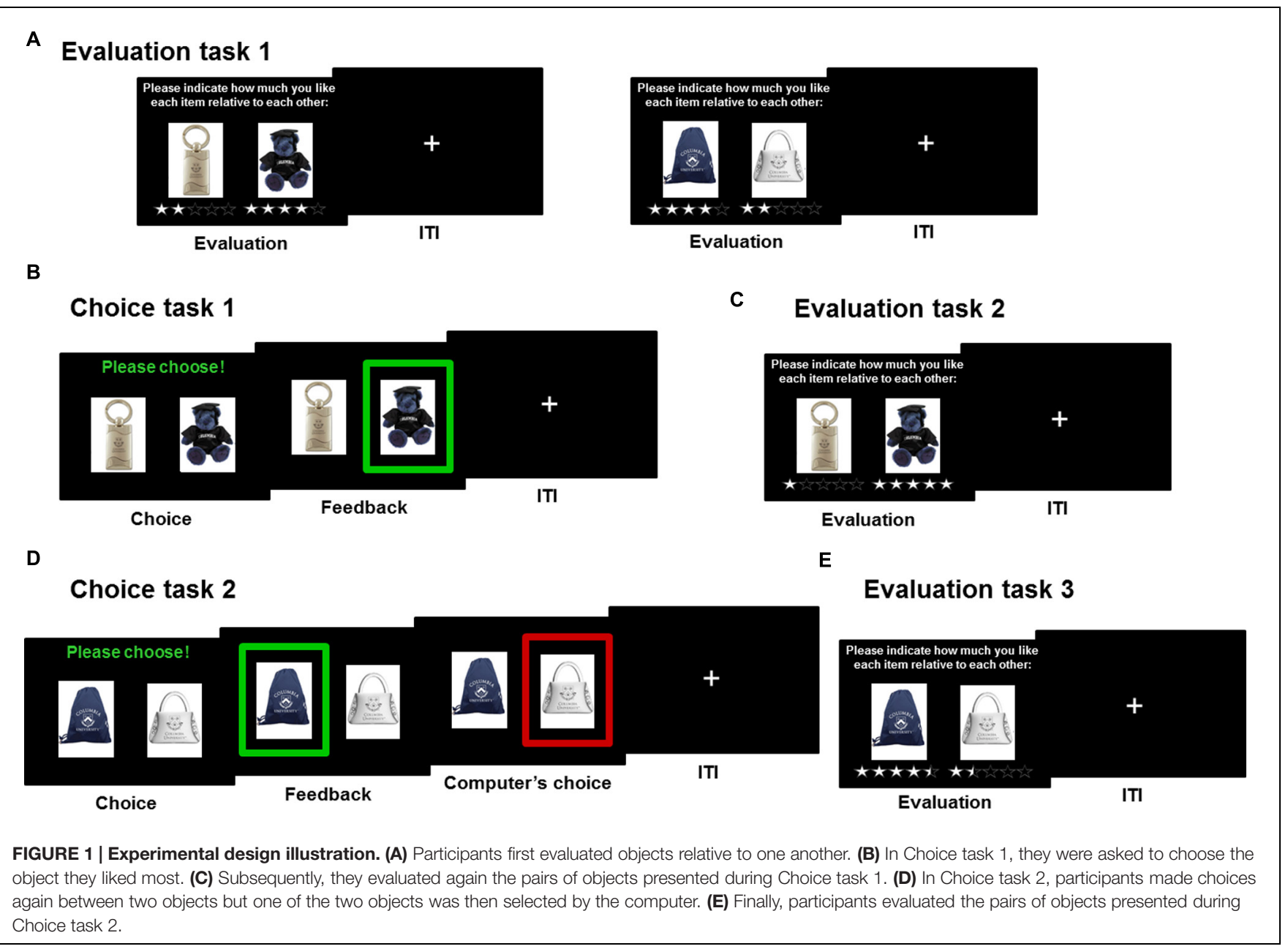

\section{Choice Task 1}

Participants were presented with 40 of the 80 pairs of objects selected in Evaluation task 1, presented in a random order. On each trial, they were asked to make spontaneous decisions and were given $3 \mathrm{~s}$ to choose the object they would most prefer obtaining at the end of the experiment. Their selection was then highlighted by a green frame for $2 \mathrm{~s}$ and the trial ended with a $1-5 \mathrm{~s}$ jittered ITI (Figure 1B). Importantly, participants were instructed that at the end of the experiment, one of the selected objects throughout the experiment would be randomly picked by the computer and given to them.

\section{Evaluation Task 2}

Participants were then asked to re-evaluate these 40 pairs of items, like they did in Evaluation task 1 (Figure 1C). All pairs of objects were presented in a random order.

\section{Choice Task 2}

The procedure was identical to Choice task 1, the only exception being that after making their choice, a red frame appeared on the screen for $2 \mathrm{~s}$ (Figure 1D). Participants were told that this task was synchronized with the other participant and that on each trial the computer would randomly select either their own choice, or that of the other participant. Importantly, participants were instructed to pay close attention to the objects highlighted in red because the object they would receive at the end of the experiment would be randomly selected among the objects they selected during Choice task 1 or amongst the items highlighted in red during Choice task 2. In reality, the tasks were not synchronized and the choices highlighted in red were pre-determined so that half the time the feedback would be congruent with the participant's choice, and half the time, it would be incongruent with their choice.

This task consisted of 80 trials, including the 40 pairs of objects used in Choice task 1 and the remaining 40 pairs of objects selected after Evaluation task 1. They were all presented in a random order. Note that the change in evaluation did not differ between the pairs of objects used in both Choice tasks versus the pairs of objects used in Choice task 2 only [paired $t$-test, $t(27)=0.22, p=0.89$ ], thus further analyses do not distinguish between these pairs of objects.

Note that the order of the sessions and the frame color highlighting the participant's selection were not counterbalanced across subjects and choice tasks, which might be potential limitations to the current design. 


\section{Evaluation Task 3}

Participants were asked to re-evaluate the pairs of objects used in Choice task 2, like they did in previous Evaluation tasks (Figure 1E). All pairs of objects were presented in a random order.

\section{Quantifying evaluation change}

For each pair of objects presented, the evaluation of one object was made relative to the other object (i.e., an increase in the evaluation of one object necessarily implied a decrease in the evaluation of the other object). Therefore, we only report the evaluation change for the chosen object.

\section{Evaluation Change Elicited by Choice Task 1}

For each trial $t$, Evaluation Change Scores for Choice task 1 (ECS1) were computed as follows:

ECS-1 $(t)=$ rating from Evaluation task 2 for chosen object

$(t)$ - rating from Evaluation task 1 for chosen object $(t)$.

To create an ECS- 1 for each participant $P$, we averaged ECS-1 across all trials for that particular participant as follows:

ECS-1-M $(P)=$ mean $(\operatorname{ECS}-1(t))$.

\section{Evaluation Change Elicited by Choice Task 2}

Based on participants' reports from previous pilot studies, 'congruent' trials (i.e., when the computer selected the chosen object) were excluded from analyses reported in the Results section. Despite being obtainable, chosen objects in congruent trials elicited various interpretations across participants. Some participants thought that it meant that the computer had randomly elected their own choice every time while in reality it could also mean that the computer had selected the other participant's choice (which was similar to theirs). In addition, and regardless of this distinction, some participants reported being happy when the computer's choice was congruent with theirs because that meant that they still had a chance to obtain the chosen object. On the other hand, some participants reported being unsatisfied during Choice task 2 in general because they had no control over the outcome. Therefore, given the multitude of interpretations possible for congruent trials and the different cognitive processes that might have been involved during these trials, they were not considered further. Note that in the case of incongruent trials, participants interpreted the fact that their choice was not selected as the computer having selected the other participant's choice, as explained in the instructions. Thus, incongruent trials did not involve analogous possible misinterpretations.

Evaluation Change Scores for Choice task 2 (ECS-2) were computed as follows for each trial $t$ :

ECS-2 $(t)=$ rating from Evaluation task 3 for chosen object $(t)$ - rating from Evaluation task 1 for chosen object $(t)$.

Note that computing ECS-2(t) using the rating from Evaluation task 2 for the chosen object $(t)$ when available (that is for the choice pairs used in Choice Task 1) did not qualitatively alter our results (2x2 ANOVA, factor Evaluation change: $[F(1,55)=0.01, p=0.91]$, factor explicit self-esteem: $[F(1,55)=0.43, p=0.51]$, interaction: $[F(1,55)=5.47, p=0.02]$.

To create an ECS- 2 for each participant $P$, we averaged ECS-2 across all trials for that particular participant as follows:
ECS-2-M $(P)=$ mean $($ ECS- $2(t))$.

For completeness, we conducted similar analyses to the one reported in the Results section replacing ECS-1-M with either (1) the congruent trials from Choice task 2 alone, or replacing it with (2) ECS-1-M + congruent trials from Choice task 2. The results from these 2x2 ANOVAs did not qualitatively differ from the analysis reported in Section "Results" and the interaction between the factors Evaluation change and explicit self-esteem remained virtually significant in both analyses Analysis 1: no main effect for the factor Evaluation change $[F(1,55)=0.74, p=0.39]$ or the factor explicit self-esteem $[F(1,55)=2.9, p=0.09]$, interaction between these two factors $[F(1,55)=3.55, p=0.06]$; Analysis 2: no main effect for the factor Evaluation change $[F(1,55)=0.36$, $p=0.55]$ or the factor explicit self-esteem $[F(1,55)=0, p=0.97]$, interaction between these two factors $[F(1,55)=3, p=0.08]$.

\section{Statistical Analysis}

All statistical tests were performed using Matlab 7.9.0 (R2009b). The linear regression modeling the relationship between the evaluation of chosen objects (dependent variable) and explicit self-esteem (independent variable) was performed using the corrcoef function. The $2 \times 2$ ANOVA with the factors Evaluation change (ECS-1-M versus ECS-2-M) and explicit self-esteem (High versus Low self-esteem) was performed using the anova 2 function. One-sample $t$-tests were performed using the ttest function, and two-sample $t$-tests were performed using the ttest 2 function. Note that all statistical tests are two-tailed unless stated otherwise.

\section{RESULTS}

As predicted, the evaluation of chosen objects that were obtainable (ECS-1-M) was enhanced as a function of explicit self-esteem (linear regression; $r=0.45, p<0.05$ ). Additionally, we conducted a $2 \times 2$ ANOVA with the factors Evaluation change (ECS-1-M versus ECS-2-M) and explicit self-esteem (High versus Low self-esteem) to test for differences between participants with high versus low explicit self-esteem in their tendency to enhance their evaluation of chosen objects depending on their obtainability. A median split was used to create the High selfesteem group (14 participants) and the Low self-esteem group (14 participants). This analysis revealed no main effect for the factor Evaluation change $[F(1,55)=0.03, p=0.87]$ or the factor explicit self-esteem $[F(1,55)=0.77, p=0.38$; Figure 2]. As expected, we found a significant interaction between these two factors $\left[F(1,55)=6.75, p=0.01, \eta_{\mathrm{p}}^{2}=0.11\right]$, such that participants in the high self-esteem group exhibited more Evaluation change for chosen objects that were obtainable compared to chosen objects that were unobtainable $[M=0.19, S D=0.33, t(13)=2.13$, $p=0.05, d=0.77]$ whereas participants in the low self-esteem group exhibited more evaluation change for chosen objects that were unobtainable compared to those that were obtainable $[M=0.17, S D=0.23, t(13)=2.67, p=0.02, d=0.59]$.

Interestingly, when asked in a post-experiment questionnaire whether they felt that their evaluations had changed following either Choice task, participants reported that their relative 


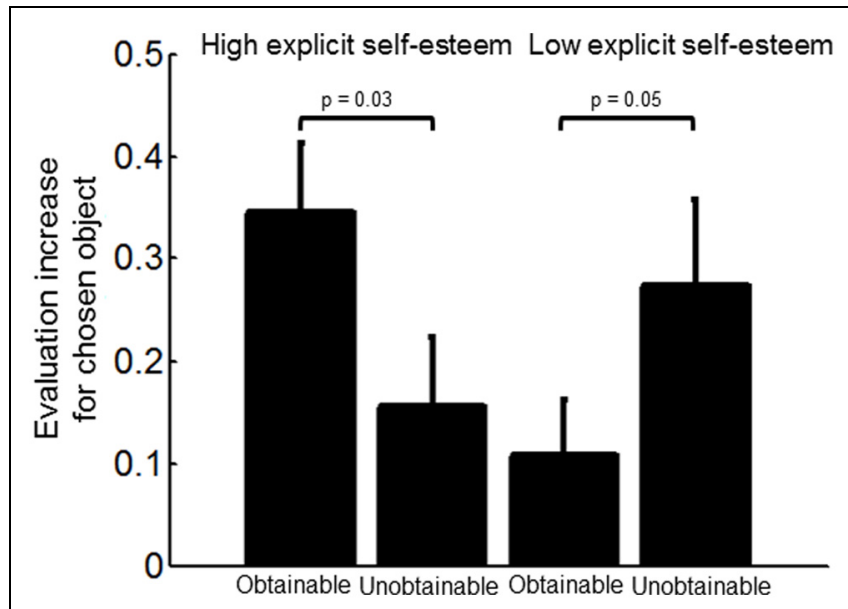

FIGURE 2 | Interaction between the factors evaluation change of chosen objects according to their obtainibility (ECS-1-M versus ECS-2-M) and explicit self-esteem (Low versus High group; 2x2 ANOVA). Participants in the high explicit self-esteem group showed more evaluation change of chosen objects when they were obtainable [one-tailed paired $t$-test, $t(26)=2.02, p=0.03$ ] while those in the low explicit self-esteem group showed increased preference for chosen objects that were unobtainable [one-tailed paired $t$-test, $t(26)=-1.67, p=0.05$ ]. Error bars represent SEM.

evaluation for the chosen object remained unchanged following Choice task $1[M=5.46, S D=1.73, t(27)=1.42, p=0.17$, one sample $t$-test comparing the mean to the neutral value 5 on our 1-9 Likert scale] and Choice task $2[M=5, S D=1.72, t(27)=0$, $p=1$, one sample $t$-test comparing the mean to the neutral value 5 on our 1-9 Likert scale]. This suggests that evaluation change occurred without participants' awareness, and regardless of the obtainability of the chosen object.

\section{DISCUSSION}

The present results provide preliminary evidence indicating that obtainability of chosen objects differentially impacts the evaluation of these objects according to one's sense of explicit selfesteem. Specifically, participants with high self-esteem increased their relative evaluation of chosen objects further when they may be obtained in the future as compared to when they were unobtainable, whereas participants with low self-esteem showed the reverse pattern.

First, we provide evidence suggesting that explicit self-esteem modulates evaluations of chosen objects that are obtainable in a self-serving way, extending prior findings demonstrating a similar modulation by implicit self-esteem (Gawronski et al., 2007). This finding accords well with the idea that the act of choosing an object that we may later belong to the self generates an association between this object and the self, giving rise to a transfer of self-evaluation to the chosen object, a phenomenon referred to as associative self-anchoring (Greenwald and Banaji, 1995; Cadinu and Rothbart, 1996; Walther and Trasselli, 2003; Gawronski et al., 2007). Consistent with the idea that associative self-anchoring is independent from dissonance reduction processes (Gawronski and Bodenhausen, 2006; Gawronski et al., 2007), all objects within each pair of objects used in the current study were differently valued and thus, should not elicit cognitive dissonance (Festinger, 1957; HarmonJones and Mills, 1999). Indeed, it has long been argued that having to choose between equally attractive alternatives generates cognitive dissonance which will be reduced through a spreadingof-alternatives effect, whereby positive features of the chosen alternative and negative features of the rejected alternative are emphasized (Brehm, 1956; Frey, 1986; Olson and Stone, 2005), an effect that has not received support in the case of differently valued alternatives (Brehm, 1956; Sharot et al., 2009; Kitayama et al., 2013).

Furthermore, our findings might relate to psychological reactance theory which, applied to the current case, would predict that eliminating the possibility of receiving a chosen object increases the attractiveness of this object (Brehm, 1966; Brehm and Brehm, 1981). Consistent with previous findings showing a negative relationship between explicit self-esteem and reactant behavior (Joubert, 1990), we found that participants with low explicit self-esteem exhibited enhanced evaluations for chosen objects that were unobtainable compared to objects that were obtainable, an effect that was reversed in participants with high explicit self-esteem. Note that in the case of threat to freedom (as opposed to elimination of freedom as is the case in the current study), self-esteem has been shown to correlate positively with reactant behavior (Brockner and Elkind, 1985); however it is not surprising that the relationship between self-esteem and psychological reactance reverses depending on whether there is a possibility for freedom restoration.

One limitation of the current research is the relatively small sample size $(n=28)$ which leads our results to be preliminary. The fact that explicit self-esteem was measured at the end, rather than at the beginning of the experiment, places another potential limitation. Indeed, performing the task might have somehow modulated participants' sense of self-esteem, resulting in a measure of state explicit self-esteem rather than trait explicit self-esteem. However, given that the questionnaire explicitly examines trait self-esteem, and that the paradigm should not lower self-esteem at least to the capacity where it would drive the robust results presented here, we expect that any state effect to be negligible. Also, we cannot rule out the possibility that some of the choices might have been surprising to the participant. However, given that (1) choices are subjective in nature, (2) the two participants performing the experiment simultaneously did not know each other, and (3) the items we used in the experiment were all within a narrow price range, we believe that suspicions were kept to a minimum and did not interfere with the purpose of the experiment. Finally, it is important to note that the social impact of decisions (i.e., other's rejection of an item) may impact one's preferences and future studies should probe the effects of social or any other bias.

Linking our findings to positive illusions, participants with high self-esteem distorted the perception of chosen objects in a positive light compared to participants with low self-esteem, such that the relative attractiveness of these objects was enhanced 
further when there was a possibility for these objects to belong to them (Taylor and Brown, 1988). It is interesting to speculate that the rationalization deficiency exhibited by people with low selfesteem together with their tendency to ruminate over negative consequences might relate to the well-known correspondence between low self-esteem, depression and anxiety (Sowislo and Orth, 2013).

However, it is important to note that in accordance with the consistency bias where past attitudes are incorrectly remembered to resemble present's attitudes, participants' reports suggest that they were not aware of the fact that their evaluations changed between the different evaluation tasks. Consistent with the fact that participants were not aware of altering their evaluations, preference change has been shown to be an implicit process (Sharot et al., 2010; Jarcho et al., 2011; Coppin et al., 2012, 2014), an idea further exemplified by studies conducted in monkeys, children and amnesic patients (Lieberman et al., 2001; Egan

\section{REFERENCES}

Brehm, J. W. (1956). Postdecision changes in the desirability of alternatives. J. Abnormal Soc. Psychol. 52, 384-389. doi: 10.1037/h00 41006

Brehm, J. W. (1966). "A theory of psychological reactance," in Organization Change: A Comprehensive Reader, eds W. W. Burke, D. G. Lake, and J. V. Paine (New York, NY: Academic Press), 377-389.

Brehm, S. S., and Brehm, J. W. (1981). Psychological Reactance: A Theory of Freedom and Control. London: Academic Press, Inc.

Brockner, J., and Elkind, M. (1985). Self-esteem and reactance: further evidence of attitudinal and motivational consequences. J. Exp. Soc. Psychol. 21, 346-361. doi: 10.1016/0022-1031(85) 90035-6

Cadinu, M. R., and Rothbart, M. (1996). Self-anchoring and differentiation processes in the minimal group setting. J. Pers. Soc. Psychol. 70, 661-677. doi: 10.1037/0022-3514.70.4.661

Chen, M. K., and Risen, J. L. (2010). How choice affects and reflects preferences: revisiting the free-choice paradigm. J. Pers. Soc. Psychol. 99, 573-594. doi: $10.1037 / \mathrm{a} 0020217$

Coppin, G., Delplanque, S., Bernard, C., Cekic, S., Porcherot, C., Cayeux, I., et al. (2014). Choice both affects and reflects preferences. Q. J. Exp. Psychol. 67, 1415-1427. doi: 10.1080/17470218.2013.863953

Coppin, G., Delplanque, S., Porcherot, C., Cayeux, I., and Sander, D. (2012). When flexibility is stable: implicit long-term shaping of olfactory preferences. PLoS ONE 7:e37857. doi: 10.1371/journal.pone.00 37857

Egan, L. C., Bloom, P., and Santos, L. R. (2010). Choice-induced preferences in the absence of choice: evidence from a blind two choice paradigm with young children and capuchin monkeys. J. Exp. Soc. Psychol. 46, 204-207. doi: 10.1016/j.jesp.2009.08.014

Egan, L. C., Santos, L. R., and Bloom, P. (2007). The origins of cognitive dissonance: evidence from children and monkeys. Psychol. Sci. 18, 978-983. doi: $10.1111 /$ j.1467-9280.2007.02012.x

Festinger, L. (1957). A Theory of Cognitive Dissonance. Evanston: Row, Peterson \& Company.

Frey, D. (1986). "Recent research on selective exposure to information," in Advances in Experimental Social Psychology, ed. L. Berkowitz, Vol. 19 (New York: Academic Press), 41-80.

Gawronski, B., and Bodenhausen, G. V. (2006). Associative and propositional processes in evaluation: an integrative review of implicit and explicit attitude change. Psychol. Bull. 132, 692-731. doi: 10.1037/0033-2909.13 2.5.692

Gawronski, B., Bodenhausen, G., and Becker, A. P. (2007). I like it, because I like myself: associative self-anchoring and post-decisional change of implicit et al., 2007, 2010). Although some of the dissonance studies cited above suffered from the artifact raised by Chen and Risen (2010) and should be interpreted with caution, there is emerging evidence that the change in evaluation we observe might reflect a non-conscious mechanism serving important adaptive purposes. Indeed, being contented with what might be part of one's environment, rather than dwelling upon what could have been might act as a positive reinforcement loop, whereby associative self-anchoring induces a positive feeling that further enhances self-esteem, and ultimately well-being (Taylor and Brown, 1988).

\section{ACKNOWLEDGMENTS}

We thank Jessica Mizzi for assistance in running participants. This work was funded by a start-up grant to DM.

evaluations. J. Exp. Soc. Psychol. 43, 221-232. doi: 10.1016/j.jesp.2006. 04.001

Gawronski, B., and Strack, F. (2004). On the propositional nature of cognitive consistency: dissonance changes explicit, but not implicit attitudes. J. Exp. Soc. Psychol. 40, 535-542. doi: 10.1016/j.jesp.2003. 10.005

Greenwald, A. G., and Banaji, M. R. (1995). Implicit social cognition: attitudes, self-esteem, and stereotypes. Psychol. Rev. 102, 4-27. doi: 10.1037/0033295X.102.1.4

Harmon-Jones, E., and Mills, J. (1999). Cognitive Dissonance: Progress on a Pivotal Theory in Social Psychology. Washington, DC: Braun-Brumfield. doi: 10.1037/10318-000

Izuma, K., Matsumoto, M., Murayama, K., Samejima, K., Sadato, N., and Matsumoto, K. (2010). Neural correlates of cognitive dissonance and choiceinduced preference change. Proc. Natl. Acad. Sci. U.S.A. 107, 22014-22019. doi: 10.1073/pnas.1011879108

Izuma, K., and Murayama, K. (2013). Choice-induced preference change in the free-choice paradigm: a critical methodological review. Front. Psychol. 4:41. doi: 10.3389/fpsyg.2013.00041

Jarcho, J. M., Berkman, E. T., and Lieberman, M. D. (2011). The neural basis of rationalization: cognitive dissonance reduction during decisionmaking. Soc. Cogn. Affect. Neurosci. 6, 460-467. doi: 10.1093/scan/n sq054

Joubert, C. E. (1990). Relationship among self-esteem, psychological reactance, and other personality variables. Psychol. Rep. 66(3Pt 2), 1147-1151. doi: 10.2466/pr0.1990.66.3c.1147

Kitayama, S., Chua, H. F., Tompson, S., and Han, S. (2013). Neural mechanisms of dissonance: an fMRI investigation of choice justification. Neuroimage 69, 206-212. doi: 10.1016/j.neuroimage.2012. 11.034

Lieberman, M. D., Ochsner, K. N., Gilbert, D. T., and Schacter, D. L. (2001). Do amnesics exhibit cognitive dissonance reduction? The role of explicit memory and attention in attitude change. Psychol. Sci. 12, 135-140. doi: 10.1111/14679280.00323

Olson, J. M., and Stone, J. (2005). "The influence of behavior on attitudes," in Handbook of Attitudes and Attitude Change, eds D. Albarracin, B. T. Johnson, and M. P. Zanna (Mahwah, NJ: Erlbaum), 223-271.

Qin, J., Kimel, S., Kitayama, S., Wang, X., Yang, X., and Han, S. (2011). How choice modifies preference: neural correlates of choice justification. Neuroimage 55, 240-246. doi: 10.1016/j.neuroimage.2010. 11.076

Robins, R. W., Hendin, H. M., and Trzesniewski, K. H. (2001). Measuring global self-esteem: construct validation of a single-item measure and the Rosenberg self-esteem scale. Pers. Soc. Psychol. Bull. 27, 151-161. doi: $10.1177 / 0146167201272002$ 
Sharot, T., De Martino, B., and Dolan, R. J. (2009). How choice reveals and shapes expected hedonic outcome. J. Neurosci. 29, 3760-3765. doi: 10.1523/JNEUROSCI.4972-08.2009

Sharot, T., Fleming, S. M., Yu, X., Koster, R., and Dolan, R. J. (2012). Is choiceinduced preference change long lasting? Psychol. Sci. 23, 1123-1129. doi: $10.1177 / 0956797612438733$

Sharot, T., Velasquez, C. M., and Dolan, R. J. (2010). Do decisions shape preference? Evidence from blind choice. Psychol. Sci. 21, 1231-1235. doi: $10.1177 / 0956797610379235$

Sowislo, J. F., and Orth, U. (2013). Does low self-esteem predict depression and anxiety? A meta-analysis of longitudinal studies. Psychol. Bull. 139, 213-240. doi: $10.1037 / \mathrm{a} 0028931$

Taylor, S. E., and Brown, J. D. (1988). Illusion and well-being: a social psychological perspective on mental health. Psychol. Bull. 103, 193-210. doi: 10.1037/00332909.103.2.193
Walther, E., and Trasselli, C. (2003). I like her, because I like myself: self-evaluation as a source of interpersonal attitudes. Exp. Psychol. 50, 239-246. doi: 10.1026//1618-3169.50. 4.239

Conflict of Interest Statement: The authors declare that the research was conducted in the absence of any commercial or financial relationships that could be construed as a potential conflict of interest.

Copyright (c) 2016 Prévost, Bolger and Mobbs. This is an open-access article distributed under the terms of the Creative Commons Attribution License (CC BY). The use, distribution or reproduction in other forums is permitted, provided the original author(s) or licensor are credited and that the original publication in this journal is cited, in accordance with accepted academic practice. No use, distribution or reproduction is permitted which does not comply with these terms. 